\title{
Retinopathy in chronic hepatitis C patients during interferon treatment with ribavirin
}

\author{
K Jain, W C Lam, S Waheeb, Q Thai, J Heathcote
}

\begin{abstract}
Aim-To assess the ocular effect of interferon alfa $2 b$ prescribed with ribavirin in patients undergoing therapy for chronic hepatitis C.

Methods-19 patients with chronic hepatitis $C$ who satisfied the follow up criteria were assessed for ocular complications using slit lamp biomicroscopy and indirect ophthalmoscopy before, during, and after the treatment at regular intervals. Results-8/19 patients, while on treatment, developed an asymptomatic retinopathy. Among these $3 / 8$ were relapsers and 5/9 were non-responders to interferon monotherapy. All retinal changes faded, often while the patients continued the therapy. There was no significant association in occurrence of retinopathy with haematological and/or biochemical changes.

Conclusion-Retinopathy was more common in interferon monotherapy nonresponders than relapsers when treated with interferon alfa $2 \mathrm{~b}$ with the addition of ribavirin. The changes were transient, disappearing while the patients were still being treated.
\end{abstract}

(Br f Ophthalmol 2001;85:1171-1173)

Interferon is a complex group of proteins, which have an antiviral, antiproliferative, and immunomodulatory activity. Ribavirin is a synthetic guanosine nucleoside analogue with antiviral properties against a range of RNA and DNA viruses ${ }^{1}$ as well as immunoregulatory activity. ${ }^{2}$ Ribavirin crosses the blood-brain barrier and its concentration in the central nervous system is $70 \%$ or more of that in plasma. Chronic infection with hepatitis C virus is estimated to affect almost four million people in the USA, five million in Europe, and 170 million individuals worldwide. ${ }^{34}$ Only $15-20 \%$ of patients with chronic hepatitis C will have a sustained virological response to interferon therapy, ${ }^{56}$ but the combination of ribavirin with interferon increases the sustained virological response twofold in those who are interferon naive and those who are relapsers to interferon monotherapy. ${ }^{7}$ Retinal complications with interferon have been reported in the Japanese literature. ${ }^{8}$ We describe the retinal complications associated with ribavirin and interferon therapy among a nonJapanese population in this study.

Patients and methods

This was a prospective study. Of the 24 patients enrolled for ocular assessment only 19 satisfied the follow up criteria. They ranged in age from 37 to 58 years; 13 were male and six female. One was diabetic, one was hypertensive, and one had both. Seventeen patients had had interferon monotherapy before this study for the treatment of their chronic hepatitis C. Among the 19 patients eight were relapsers, nine were non-responders to interferon monotherapy, and two were interferon naive. All 19 patients were in a randomised trial of 24 weeks' (11 patients) or 48 weeks' (eight patients) duration to determine the efficacy and safety of interferon alfa $2 \mathrm{~b}$ plus ribavirin compared with those receiving interferon and placebo in the treatment of hepatitis $\mathrm{C}$ virus chronic infection. Pretreatment, all patients were positive for serum HCV-RNA by polymerase chain reaction (PCR), had chronic hepatitis $\mathrm{C}$ on liver biopsy, and elevated serum amino transferase levels for at least 6 months. Entry haemoglobin values had to be at least $120 \mathrm{~g} / \mathrm{l}$ in women and $130 \mathrm{~g}$ or more for men. Patients with evidence of decompensated liver disease were excluded from the study. Patients with HIV-1 infection or hepatitis B coinfection, previous organ transplantation, pre-existing psychiatric conditions, seizure disorder, cardiovascular disease, haemoglobinopathy, haemophilia, poorly controlled diabetes, renal failure, and autoimmune diseases were all excluded.

Patients were given either a combination of interferon alfa $2 \mathrm{~b}$ plus ribavirin for 24 or 48 weeks or interferon alfa $2 \mathrm{~b}$ plus placebo for the same period. All patients received interferon alfa $2 \mathrm{~b}$ (Intron A, Schering Plough, Kenilworth, NJ, USA) at a dose of 3 million units given subcutaneously three times a week for 24 or 48 weeks. Ribavirin (Rebetol, Schering Plough, Kenilworth, NJ, USA) or a matched placebo was given orally twice a day to a total dose of $1000 \mathrm{mg}$ (body weight less than $75 \mathrm{~kg}$ ) or $1200 \mathrm{mg}$ (body weight more than $75 \mathrm{~kg}$ ) per day. Both drugs were started and stopped at the same time. All patients were assessed for safety, tolerance, and efficacy at the end of weeks $1,2,4,6$, and 8 and every 4 weeks during the treatment. After treatment was completed, patients were followed up on weeks 4 , 8,12 , and 24 . The primary end point was sustained loss of detectable HCV-RNA at 24 weeks after the treatment.

All the 19 patients were prescribed ribavirin and interferon alfa $2 \mathrm{~b}$. Eleven patients were treated for 24 weeks and eight for 48 weeks. Ophthalmological examinations were carried out before the start of treatment and every month after initiation of treatment until completion of treatment or until the retinopathy disappeared. The examinations include: Snellen visual acuity, slit lamp biomicroscopy, intraocular pressure with applanation 
tonometry, post dilatation binocular indirect ophthalmoscopy, and assessment of posterior pole using $90 \mathrm{D}$ lens. Fundus photographs were taken for documentation and comparison when retinal abnormalities were detected.

\section{Results}

The ocular examinations were normal except for the retinopathy during the treatment period. The cotton wool spots were seen in the majority of patients and some had flameshaped haemorrhages. The background diabetic retinopathy in one patient remained stable. The retinopathy was noted 4 to 28 weeks after initiation of the treatment and disappeared in a 4-12 week period. All the patients were asymptomatic and the retinopathy disappeared in all patients who continued the treatment except one. In this patient retinopathy appeared as flame-shaped retinal haemorrhage in both eyes, which disappeared in 8 weeks followed by cotton wool patches in both eyes which disappeared 4 weeks after the completion of treatment. In all, 8/19 patients developed the retinopathy (Table 1). Among these $3 / 8$ were interferon relapsers and 5/9 were interferon non-responders. Among the nine non-responders to interferon monotherapy, who received combined therapy with interferon and ribavirin during the randomised trial period, three patients showed sustained loss of detectable HCV-RNA; among these, one patient developed retinopathy. One patient became negative for serum HCV-RNA at the 12 week period but relapsed when the treatment stopped (relapser) and also developed retinopathy. Five patients remained positive for serum HCV-RNA (remained nonresponders) of which three patients developed retinopathy.

Of the eight relapsers to interferon monotherapy who received combined therapy with interferon and ribavirin during the randomised trial period, four showed sustained loss of detectable HCV-RNA, of which one developed retinopathy, and four became negative for HCV-RNA at the 12-16 week period but relapsed later when the treatment stopped (remained relapsers); two of these developed retinopathy. Of the eight patients (four nonresponders and four relapsers) treated for 48 weeks, seven showed sustained loss of serum

Table 1 Retinopathy findings (ribavirin and interferon)

\begin{tabular}{ll}
\hline Number of patient & 8 \\
Age (years) (range) & $37-56$ \\
Sex (males/females) & $7 / 1$ \\
Risk factors for retinopathy: & \\
(a) Diabetes mellitus & 0 \\
(b) Hypertension & 0 \\
(c) Both (a+b) & 1 \\
(d) None & 7 \\
Retinopathy: & \\
(a) Cotton wool spots & 6 \\
(b) Retinal haemorrhage & 1 \\
Both (a)+(b) & 1 \\
Retinopathy at: & \\
Baseline & 0 \\
4 Weeks & 2 \\
8 Weeks & 1 \\
16 Weeks & 2 \\
20 Weeks & 2 \\
28 Weeks & 1 \\
Duration of retinopathy & $(4-12$ weeks) \\
\hline
\end{tabular}

HCV-RNA (negative) by 16 weeks, one remained positive for HCV-RNA, and only two showed retinopathy (one relapser and one non-responder). Of the 11 patients (two naive, five non-responders, and four relapsers) treated for 24 weeks, four remained nonresponders (HCV-RNA positive), one naive patient became a non-responder, one naive patient became a relapser, four relapsers remained as relapsers, one non-responder became a relapser, and six patients developed retinopathy, of which four were nonresponders and two were relapsers. None of the naive patients showed any retinopathy.

The haemoglobin, platelet, and leucocyte counts did not show any significant difference with or without retinopathy in these patients. Although in most cases these values decreased from the screening value during the first 4-8 weeks and reached a nadir, they remained in the near normal range. Serum aminotransferase levels decreased from the screening values within the first 4-8 weeks of the start of therapy in all these patient. There was no significant association between the haematological or the biochemical values with the onset of retinopathy.

\section{Discussion}

Interferon associated retinopathy was first recognised in 1990 when Ikeb and associates ${ }^{9}$ reported a 39 year old patient with retinal haemorrhages and cotton wool spots following intravenous administration of interferon. The reported incidence ${ }^{10}$ of interferon related retinopathy in the literature varies from $18-86 \%$. This variability could be related to several factors including the dose of interferon, associated systemic conditions, frequency of the eye examination, and the presence of underlying retinal vascular disease. The exact mechanism of interferon retinopathy is not known but it is thought to be related to the disturbance in retinal microcirculation. ${ }^{11}$ Ribavirin, when used in combination with interferon, ${ }^{12-14}$ is suggested to have synergistic action and offer sustained biochemical, histological, and virological response $^{15-18}$ over interferon monotherapy in patients treated for chronic hepatitis C. The ocular side effects of ribavirin include a mild watery eye and conjunctivitis, which were not seen in this study.

The interferon induced retinopathy includes cotton wool spots and superficial retinal haemorrhages that are usually located in the posterior pole around the optic nerve head. These retinal lesions in our patients were asymptomatic and reversible. Symptoms such as irritation, pain, and decreased visual activity have been reported with interferon therapy. Atypical retinal and ocular side effects have also been reported with interferon, including branch retinal artery occlusion, ${ }^{19}$ retinal detachment, ${ }^{20}$ subconjunctival haemorrhage, ${ }^{21}$ optic disc oedema, neovascular glaucoma, vitreous haemorrhage, and panophthalmitis. ${ }^{22}$ These lesions have been reported in a small number of patients and may represent coincidence rather than a true association with interferon use. There are also reports of retinopathy 
in untreated patients with hepatitis $\mathrm{C}^{23}{ }^{24}$ which are similar to those described in interferon retinopathy. This was not observed in any of our patients (pretreatment).

None of our patients developed any of the atypical ocular finding or visual symptoms. Their visual acuity remained unchanged throughout the course of the treatment. The retinal findings were often subtle with the few scattered cotton wool spots and small retinal haemorrhages in the posterior pole. The retinopathy occurred by 16 weeks in the majority $(62 \%)$ of patients after initiation of combination therapy and disappeared in the majority $(87.5 \%)$ of cases in the $4-8$ week period while the patients continued to receive the treatment. This suggests that treatment can be continued in the presence of retinopathy. The retinopathy disappeared in all when the treatment was stopped or immediately thereafter.

The data in this study suggest that there is no correlation of occurrence of retinopathy with the duration of treatment as the retinopathy is seen by 16 weeks in majority of patients irrespective of the duration of treatment ( 24 or 48 weeks); the longer duration (48 weeks) of treatment did not produce any increase in retinopathy but showed absence of detectable serum HCV-RNA in 7/8 of patients. This suggests that as the combination treatment for longer duration (48 weeks) improves the hepatitis and liver function it may be protecting the patients from retinopathy, perhaps by improved metabolic regulation. Nonresponders (5/9) and relapsers (3/8) were at increased risk of developing retinopathy at early stages of treatment, again suggesting poor liver function and poor metabolic regulation of interferon, and ribavirin may be a factor contributing to the occurrence of retinopathy along with various other unknown factors; this needs to be elucidated by further study. Patients with diabetes and hypertension are at increased risk of developing retinopathy ${ }^{25}{ }^{26}$; however, this was not observed in our study. In addition, patients with poorly controlled diabetes, hypertension, autoimmune diseases, and also patients with cardiovascular diseases were excluded from treatment. Previous reports have suggested that blurred vision may occur when the platelet count decreases to less than $103 \times 10^{9} / 1$, suggesting a possible association of retinopathy with thrombocytopenia. ${ }^{27}$ In our study such an association was not observed. The other haematological and biochemical changes, which took place during therapy, did not show any relation to the onset of retinopathy.

\section{Conclusion}

Retinopathy was seen more in interferon monotherapy non-responders than relapsers and a significant reduction in serum HCVRNA seen with longer duration ( 48 weeks) of treatment with interferon and ribavirin in combination. None of the naive patient developed any retinopathy. At the present time, there are no established guidelines for the follow up of patients receiving either interferon monotherapy or interferon in combination with ribavirin treatment. Since all of the patients who developed retinopathy were asymptomatic and the retinopathies were all reversible, we recommend ophthalmic follow up of patients who are symptomatic and that treatment can be continued in the presence of retinopathy.

1 Patterson JL, Fernandez-Larson R. Molecular action of ribavirin. Rev Infect Dis 1990;12:1332-46.

2 Ning $D$, Brown D, Parodo J, et al Ribavirin inhibits viral-induced macrophage production of TNF, IL-1, the procoagulant fg 12 prothrombinase and preserves TH1 cytokine production but inhibits TH2 cytokine response. $\mathcal{F}$ Immunol 1998;160:487-93.

3 Alter MJ. Epidemiology of hepatitis C. Hepatology 1997; 26(suppl 1):62-5.

4 WHO. Hepatitis C: global prevalence. Wkly Epidemiol Rec 1997;72:341-4.

5 Poynard T, Marcellin P, Lee SS, et al. Randomized trial of interferon $\alpha 2 \mathrm{~b}$ plus ribavirin for 48 weeks or for 24 weeks versus interferon $\alpha 2 b$ plus placebo for 48 weeks for treatment of chronic infection with hepatitis $\mathrm{C}$ virus. Lancet 1998;352:1426-32.

6 Mchutchison JG, Gordon SC, Schiff ER, et al. Interferon alfa-2b alone or in combination with ribavirin as initial alfa-2b alone or in combination with ribavirin as initial
treatment for chronic hepatitis C. N Engl f Med 1998;339: treatment

7 Davis G L, Esterban-Mur R, Rustigi V, et al. Interferon alpha- $2 \mathrm{~b}$ alone or in combination with ribavirin for the treatment of relapse of chronic hepatitis C. $N \mathrm{Engl} F \mathrm{Med}$ 1998;339:1493-9.

8 Kawano T, Shigehira M, Uto H, et al. Retinal complications during interferon therapy for chronic hepatitis C. Am $\mathcal{F}$ Gastroentrol 1996;91:309-13.

9 Ikebe T, Nakatsuka K, Goto M, et al. A case of retinopathy induced by intravenous administration of interferon. Folia Ophthalmol fpn (Ganka-Kiya) 1990;41:2291-6.

10 Takikawa $H$, Kawakubo H, Yazzawa $M$, et al. Interferon associated retinopathy induced by interferon beta. Ganka 1994;36:189-93.

11 Myron Y, Ben SF. Ocular pathology: a text and atlas. 3rd ed. Philadelphia: Lippincott, 1989

12 Chemello L, Cavalletto L, Bernardinello E, et al. The effect of interferon alfa and ribavirin combination therapy in
naive patients with chronic hepatitis C. $f$ Hepatol 1995;23(Suppl 2):8-12.

13 Acero D, Adrados M, Gonzalez-Huix F, et al. Ribavirin treatment in patients with chronic hepatitis $\mathrm{C}$ refractory to interferon-alpha. Gastroenterology Hepatologis (Spanish) 1996;19:243-6.

4 Richard O, Schvarcz R, Weiland O. Therapy of hepatitis C: interferon and ribavirin. $\mathcal{F}$ Hepatol 1997;26(suppl 1):1085115.

15 Lai MY, Kao JH, Yang PM, et al. Long-term efficacy of ribavirin plus interferon alfa in the treatment of chronic hepatitis C. Gastroenterology 1996;111:1307-12.

16 Gane EJ, Tibbs CJ, Ramage JK, et al. Ribavirin therapy for hepatitis $\mathrm{C}$ infection following liver transplantation. Transplant Int 1995;8:61-4.

17 Kakumu S, Yoshioka K, Wakita $\mathrm{T}$, et al. A pilot study of ribavirin and interferon beta for the treatment of chronic ribavirin and interferon beta for the treatment

18 Schvarcz R, Ando Y, Sonnerborg A, et al. Combination treatment with interferon $\alpha 2 \mathrm{~b}$ and ribavirin for chronic hepatitis $\mathrm{C}$ in patients who have failed to achieve sustained response to interferon alone: Swedish experience. $\mathcal{f}$ Hepatol 1995;23(Suppl 2):17-21

19 Futami H, Chuman T, Naoi N, et al. Ocular complication during systemic interferon therapy. $\mathscr{J p n} \mathcal{F}$ Clin Ophthalmol (Rinsho Ganka) 1994;48:553-7.

20 Okanoue T, Sakamoto S, Itoh Y, et al. Side effects of high dose interferon therapy for chronic hepatitis C. 7 Hepatol 1996;25 283-91.

21 Hayasaks S, Fujii M, Yamamoto Y, et al. Retinopathy and subconjunctival haemorrhage in patients with chronic viral hepatitis receiving interferon alfa. Br f Ophthalmol 1995;79: 150-2.

22 Yoshitoshi T, Saegusa K, Watanabe A, et al. A case of panophthalmitis developed during interferon therapy. $\mathcal{F p n} f$ Clin Ophthalmol (Rinsho Ganka) 1994;48:692-3.

23 Abe T, Sakuragi S, Kuramitsu T, et al. Retinopathy associated with hepatitis C virus. Fpn f Clin Ophthalmol (Rinsho Ganka) 1993;47:297-300.

24 Abe T, Nakajima A, Satoh N, et al. Clinical characteristics of hepatitis C virus-associated retinopathy. $\mathcal{J p n} f \mathrm{Clin}$ Ophthalmol 1995;39:411-9.

25 Chuman T, Nao-I N, Sawada A, et al. Interferon induced retinal changes. F fpn Ophthalmol Soc (Nichigan-Kaishi) 1994;98:616-21.

26 Hayasaks S, Nagaki Y, Matsumoto M, et al. Interferon associated retinopathy. Br f Ophthalmol 1998;82:323-5.

27 Seki K, Itou Y, Nishi Y, et al. Interferon therapy for type C chronic active hepatitis and retinal hemorrhage. Acta Hepatol fpn 1993;34:385-91. 